\title{
TESTING OF COMPOSITE PANELS USED AS COMPONENTS OF A FREIGHT WAGON BY THERMOVISION
}

\author{
Andrzej WRÓBEL*, Marek PLACZEK*, Andrzej BUCHACZ* \\ *Faculty of Mechanical Engineering, Silesian University of Technology, \\ ul. Konarskiego 18A, 44-100 Gliwice, Poland
}

andrzej.wrobel@polsl.pl, marek.placzek@polsl.pl, andrzej.buchacz@polsl.pl

received 22 September 2014, revised 14 December 2015, accepted 22 December 2015

\begin{abstract}
In this paper research methods for detection of laminate panels damage were presented. The most common damage is: matrix cracking laminate interlayer damage to joints, connecting cracks, delamination and fiber breakage. The tested laminates will be used as assemblies and sub-assemblies of freight wagon. Other methods of modeling of machines by means of transducers are shown in Płaczek $(2012,2015)$ and Białas (2010). As part of the project authors were collaborated with specialists from other research centers and scientific research (Bocian and Kulisiewicz, 2013). As a part of future work the places where we will be able to replace the standard materials by parts made of laminate will be shown. Layered composites despite many advantages have also disadvantages. From last mentioned it is a relatively low resistance to transverse impact. When the laminate is used as a decorative element, its small damage is not a problem. The problems start when the composite satisfies more responsible tasks such as: is a part of the technical means for example of a railway wagon. Aspect of continuous monitoring of the technical state of the laminate is very important. Current technology provides numerous opportunities for non-destructive methods of technical inspections. In this paper method for testing of large areas, completely non-contact, based on the methods of thermography, was presented. It consists heating by using the composite tubes and examining it through a thermal imaging camera. Length of heating, and consequently the temperature to which the laminate is heated mostly were chosen experimentally. During the measurements, the camera measures the intensity of radiation, not temperature. Received thermogram is not always a precise representation of the actual temperature, because the camera does not reach only the radiation from tested object, but also reaches the radiation coming from the environment and reflected objects etc. As part of the research authors also we undertook other work related to Mechanical Engineering (Wróbel et al., 2008, 2010, 2012, 2013, 2015; Płaczek et al., 2014). Cooperation with other national and European centers has contributed to many publications of authors for example Tuma et al. $(2013,2014)$ and Jamroziak and Kosobudzki (2014).
\end{abstract}

Key words: Thermovision, Components, Testing, Panels, Imaging Method

\section{PRODUCTION OF THE TEST PANELS}

There are several basic groups of laminates:

- one-sided: only one side has a certain esthetic values, and the second is not visible, such as for example a sink,

- double-sided: both sides must meet certain esthetic requirements, such as for example boat,

- standard: used for gluing different types of elements,

- postforming: used in the manufacture of kitchen worktops, or parts of furniture,

- floor: floor panels manufacture (Buchacz et al., 2013)

The method of production is selected depending on the type of laminate and technology, which possess a manufacturing plant. Hand lamination is the most popular method for the production of laminates among amateur applications. It is used a form, which shape is the negative of the expected shape. This form is prepared by polishing, afterwards the separatory layer is applied. For this purpose, several substances are used, the most popular is a polyvinyl alcohol (PVA), and various types of paste based on natural waxes. The application of PVA is easier and less time consuming, but during laminating the alcohol layer forms a very thin film, which can influence the surface structure. In case when the final element is to be perfectly smooth, it is necessary to apply the wax. This operation is time consuming because of the need to impose several layers (6-7), and requirement that, after each of layer the polishing is needed. However, in case of the reuse of the form, it is sufficient only wax "refresh", by applying one or two layers. The next step is to apply the gel coat with the addition of a hardener. It is a colored resin, which imparts an aesthetic appearance and protects the laminate center from water and sunlight. This process can be done manually by brushing, or by spraying. Partial gelation of the gel coat is followed by the resin layered application, alternating with fiber glass (alternatively fiber carbon) in a form of mat or fabric. The whole should be rolled with a special knurled roller in order to remove air bubbles. If this activity is neglected, there is a high risk of delamination. After application of a certain number of layers, hardening process occurs. After a total transition into the solid state, inner layer is covered by so-called topcoat, which performs functions similar to the gel coat layer, but is no longer not so even and smooth. The final step is to split the element from the form. The method is dependent on the imagination of the operator, usually this is done with a wooden wedge and hammer. 


\section{THERMAL IMAGING METHOD}

The thermal imaging method is used to examine large areas, completely non-contact, based on thermography methods. It involves heating by using the lamp of certain composite and examining it using a thermal imaging camera. Time of heating, and consequently the temperature to which the laminate is heated mostly were chosen experimentally. It depends inter alia on the thickness of laminate, reinforcement and matrix.

Thermal imaging is based on infrared radiation. The range can be divided into smaller bands contractually defined:

- near-infrared (0,75-3 $\mu \mathrm{m})$,

- average infrared (3-6 $\mu \mathrm{m})$,

- far infrared (6-15 $\mu \mathrm{m})$,

- very far infrared (15-100 $\mu \mathrm{m})$.

Thermographic studies generally use the far infrared range. During the measurements, the camera measures the radiation intensity, not temperature. By this, thermogram is not always an accurate representation of the actual temperature. These inaccuracies arise from the fact that the camera does not reach only the radiation from the test object, but also reaches the radiation coming from the environment and those reflected by the other surfaces, etc. On the quality of the measurement has also influence temperature, in which the tests are conducted. The higher, the more reliable the thermogram is. In case of advanced cameras are possible measurements up to $-20^{\circ} \mathrm{C}$, but while being owner of cheaper thermal imaging camera the limit temperature is about $-5^{\circ} \mathrm{C}$. In order to minimize the impact of other objects and the environment during the test, a number of camera settings should be introduced, i.e.: object emissivity, humidity, ambient temperature, the distance from an object to the camera. After introducing those information, a number of camera settings must be also changed:

- temperature range (the smaller range the more accurate differences will be seen),

- setting of the optical focal length,

- compensation of the image,

- level (expected temperature).

When there is no possibility of testing from both sides, there is a possibility to set the camera and flash from one side. In this case the way of interpretation of the results should be changed, because part of the damages in the first set is seen as stains of lower temperature, and with the other set of the camera, the same place will be seen as a stain of the higher temperature. Significant impact on the results of the research has a way of heating.

For this purpose is applied the heating:

- pulse - surface is uniformly heated by thermal pulse ,

- modulating - intensity of the heat pulse varies sinusoidally,

- pulse-phase - connection of pulse and modulation methods.

The tested element must not only be heated by lamps or radiators. For this purpose also vibrating methods are used. The tested laminate is subjected to vibrations, which through friction change the temperature (Szczepanik at al. 2008).

\section{THERMAL IMAGING TEST OF LAMINATED PANEL}

The aim of the study was to carry out non-destructive thermal imaging testing of laminate and determination of influences of the laminate properties for their analysis. The results are affected by many factors, such as frame emissivity (color and type of surface), which carries a panel, the environment, the type of lighting and form the same panel shape, method of molding).

The present work includes several variants of the study and at the final stage, it was concluded that the factors have the most significant impact on the effect of thermal imaging diagnosis. Research has characteristics of the typical comparative method, because the goal is not to designate a specific temperature, but to determine the difference in temperature between the places where damage exists, and the places where does not. Preparation of test-bench and testing was carried out in a two-person team. Three plates with dimensions of $400 \times 500 \mathrm{~mm}$ with polyester matrix were performed:

Panel no 1:

a) Type of fiber: fiberglass mat $300 \mathrm{~g} / \mathrm{m}^{2}$,

b) number of layers: 3

c) the top layer: black polyester gelcoat, polished,

d) panel thickness: 1,8 mm,

e) the type of damage:

- delamination resulting from the production process (bad rolling)

- delamination created by the stroke,

- cracks,

- bad separation of form from the element (damage of the divider layer),

- uneven distribution of gel coat,

- too strong gelcoat rolling.

Panel no. 2:

a) Type of fiber: glass roving fabric $150 \mathrm{~g} / \mathrm{m} 2$,

b) number of layers: 3

c) the top layer: black polyester gelcoat, matt,

d) panel thickness: $1,7 \mathrm{~mm}$,

e) the type of damage:

- delamination created after the stroke,

- cracks,

- uneven distribution of the gel coat.

Panel no 3:

a) Type of fiber: fiberglass mat $300 \mathrm{~g} / \mathrm{m} 2$,

b) number of layers: 4 ,

c) the top layer: No gelcoat,

d) board thickness: $1,6 \mathrm{~mm}$,

e) the type of damage:

- stratification resulting from the production process (bad rolling)

- delamination created after the stroke,

- cracks,

- local saturation and the unsaturation of the resin.

While preparing panels intentional errors were done. They should copy damages, that most frequently occur in laminates.

On the first panel, a number of defects associated with the uneven distribution of gel coat, scratches and small pores on the gel coat layer, formed due to poor separation of the form, are shown. Photo of the panel no. 1 from the back of the panel shows a diagonal shallow crack, some delamination and local unsaturation with resin. On the front side of the panel no 2 is not visible any defect with the naked eye, but on the back side can be seen one small delamination, from which propagate cracks. In addition, 
the connections of fabric are visible and piece of the loose fiber, which was laminated as an imitation of another layer. The first panel has a shiny structure, while the second was matted by sandpaper with grit of P400. Panel no. 3 does not contain a gel coat layer, which imparts gloss, but the resin itself confers a semigloss structure. As a device for pulse heating halogen bulb of $500 \mathrm{~W}$ was used. It was placed in a special housing. Lamp during the measurements was either in a stand, or was held in a hand, in order to obtain different heating method. During the study a thermal imaging camera of FLIR brand, model I7 was used.

\section{RESEARCH METHODOLOGY}

The research was conducted using thermal imaging camera type "FLIR 17", the parameters of this camera are contained in Tab. 1.

Tab. 1. Camera "FLIR I7" parameters

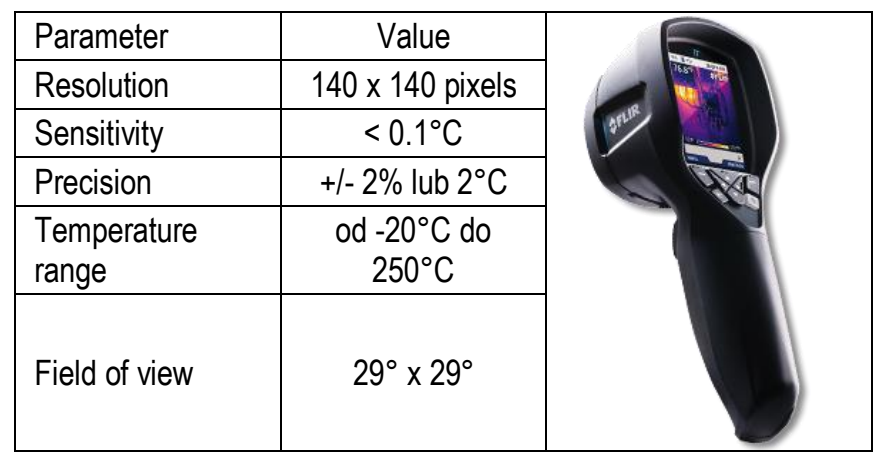

The tested samples were inserted to the black chamber made of an open cell polyurethane foam, length of 1 meter and a width of 0.5 meter, presented in Fig.1.

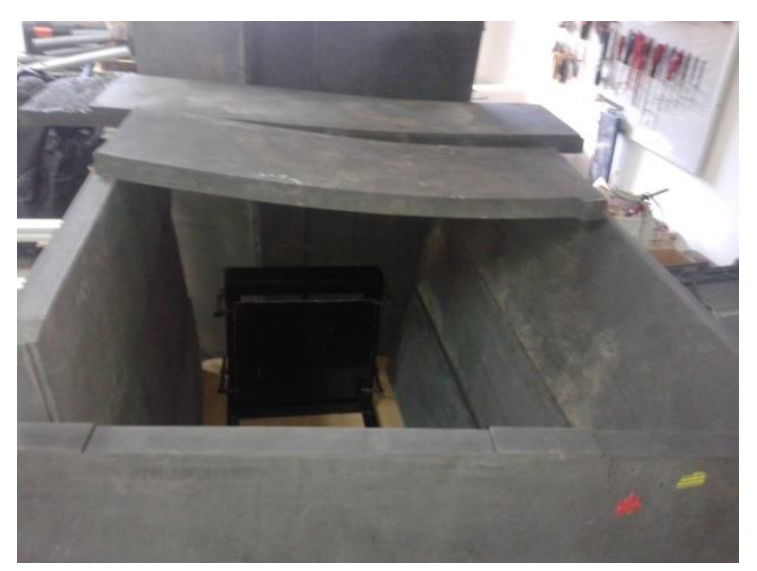

Fig. 1. The laboratory stand with tested sample placed in the black chamber

A few steps were carried out in order to eliminate measurement errors:

- a stable temperature of 21 degrees Celsius was set in the room during the measurements,

- the samples were measured in a darkened chamber (where there was no access to sunlight),

- surfaces of the samples were dry and tested samples were placed in the chamber 24 hours before testing,
- the emissivity value was exactly set.

Emissivity was calculated by using the camera. Samples were coated with a black paint with a known emissivity of 0.95 . After that the surface temperature (in painted place) using a thermal imaging camera with known emissivity was measured. Indicated temperature was the reference temperature. Then the emissivity of the camera had been setting up until the temperature measurement using the thermal imager had the same effect for the area covered and uncovered paint as paint was adjusted. So the emissivity corresponded to the emissivity of the surface plate. Experiments were repeated 4 times for each sample and the results were reproducible. Because of the large number of camera images in only a few thermograms are presented in the article.

\section{TEST RESULTS}

In case of studies with the division of laminate for parts (Fig. 2a,b.), it may be noticed more damages. Basic damage that thermography method detects is a delamination. They are visible in the case of heating from both, the front and back side, but the manner of their representation is different. If you set the lamp on the same side as the camera, delamination is seen as spots of higher temperature than the area around the damage. When the back of the laminate is heated, the thermograms show that delamination of a surface part of lower temperature. The thermogram (Fig.2) shows them as places with higher temperatures, but in addition it is surrounded by surface with lower temperature. The photos do not show the large transverse crack. It is too shallow.

a)

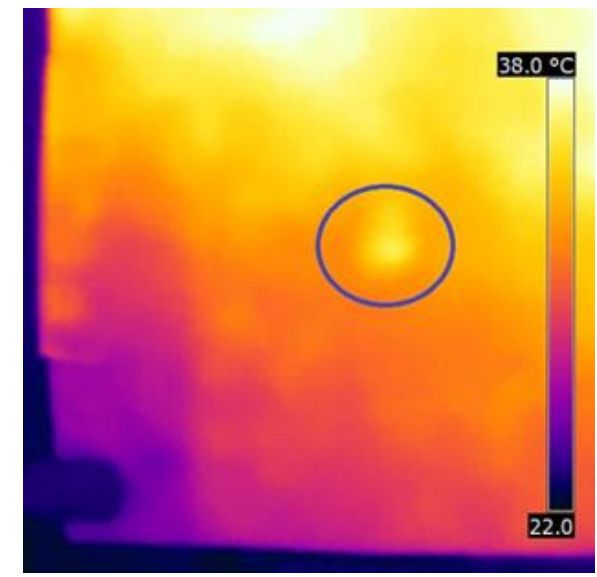

b)

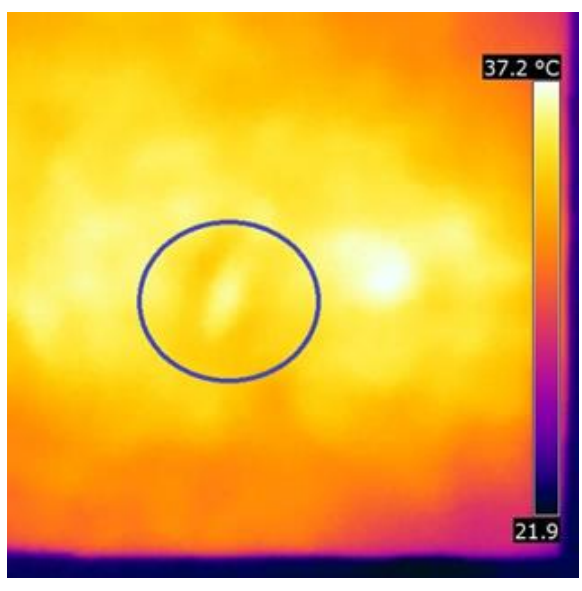

Fig. 2. The thermograms obtained while panel's testing 1: a) III quarter heated from the front, b) IV quarter heated from the front 
a)

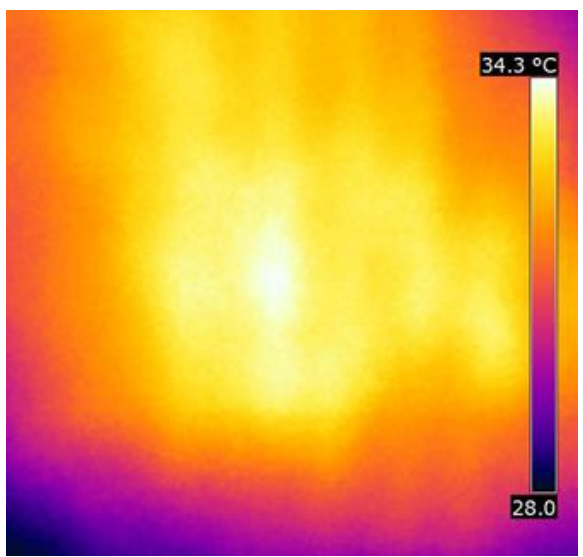

b)

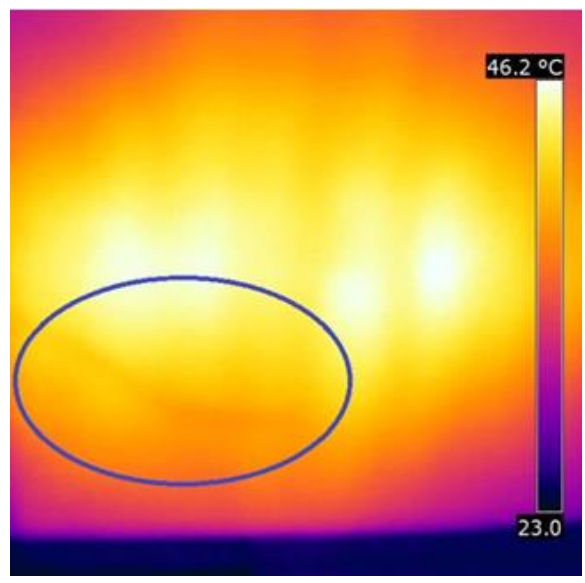

Fig. 3. The thermograms obtained through the test of panel no 2 :

a) III quarter heated from back, b) IV quarter heated from back side

The thermograms shown in Fig. 3. describing a case of studies of the panel no. 2, similarly as in the case of the laminate no 1 , the defects cannot be located by using photos of the entire panels. Defects are visible only in the case of the panel heating, divided into four parts. On the thermograms vertical stripes of lower temperature are visible. These are the effects of applying a gel coat with a brush. On the side edges of the paintbrush, band of the higher thickness of the gel coat were formed, which afterward were heated less. Furthermore, the thermogram presented on Fig. 3 b) shows a small delamination, but the cracks are already noticeable around it. In addition to these defects, the infrared method gives the feasibility of a change in a number of layers. An example of this is the thermogram of Fig. 3b) where it is clearly visible thin band running across the panel. This is part of the fiber that had to imitate change in the number of layers. Panel no. 2 was of much better quality than panel no 1 and for this reason, there is much less damage visible through the thermal camera.

On the thermograms obtained from testing of panel no 3 , contrary to appearances, and previous studies, most can be identified on the basis of photos of sub-paragraph 3. b). It can be observed on it the band of lower temperature running on the bottom. This is an area where the amount of resin was correct. However above this place, it was too much of resign. Furthermore, the laminate no 3 contained defects in the form of the local, too small saturation of resin and it is also shown on termographs depicting the entire panels. Apart from these defects, images obtained in the case of heating from the back side, show some minor delamination. Panel damage of this panel was much more, but low emission of plate made it impossible to obtain accurate results.
The thermograms obtained while heating the front side differ from those heated from the back. This is due to the difference in emissivity of the surface, because the front was shiner (smooth) than the back. The measurement results are reproducible. During the heating and cooling plates, temperature measurement error in all the samples of the same batch does not exceed $8 \%$.

a)

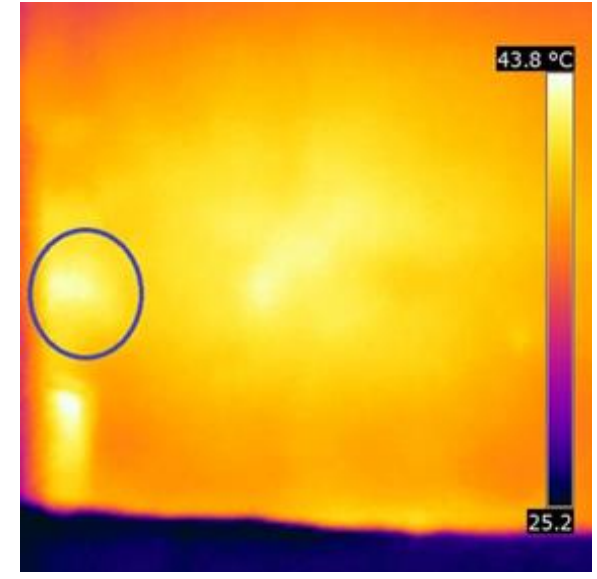

b)

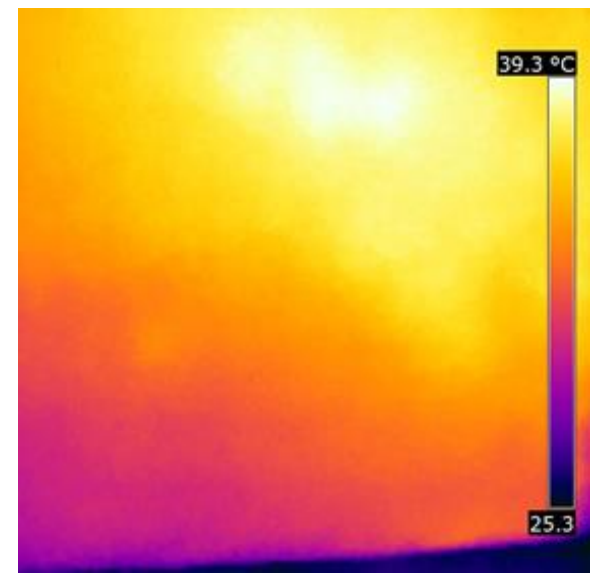

Fig. 4. The thermograms obtained with the test panel no 3: a) III quarter heated from back, b) IV quarter heated from back side

\section{SUMMARY}

Research of laminated panels by using thermal imaging method proved to be effective, because it was possible to determine the most places of damage. However, this method has many disadvantages, one of them is a method of heating. If you use an ordinary halogen lamp, the results can be affected, because the heat flux spreads evenly on the panel. In this situation the smartest solution would be to use an infrared lamp. These devices are designed specifically for uniform heating. Such devices often can be found in the painting workshops, where freshly painted parts placed on a special frame, are exposed on the action of several radiators. This speeds up the drying process, which enables faster processing. Optionally, for the heating purpose can be used larger number of lamps. Wise solution, in the case of the test panels with dimensions close to the analyzed samples, would be to use four lamps. In addition to equipment changes in heating appliances are used and changes of the heating method. For this purpose instead of pulse heating, modulation methods, which rely on oscillatory change of element heat can be used. It would not completely remove of uneven heating, but it would reduce its size. Heating 
could be also made through the excitation of the laminate vibration, but it carries behind the need of purchasing of expensive equipment. Another disadvantage of thermal imaging studies is the fact, that at the time of the study, the element must be excluded from use. Apart from this, it is important its purity, because each dirt in a form of dust, grease, etc. may cause misrepresentation of results.

On the result of thermal testing the most significant impact had not only panel's properties itself, but the manner of trials execution and the equipment used to them. Heating of a large surface area worked only in the case of panel no 3, where it was possible to notice differences in saturation of the resin. Heating from the distance resulted in a uniformly warming up of the panel, but the process required a longer duration of lamp action. With this method of heating, the entire panel, including the center heat up evenly and it was not possible to locate the places of damage.

In the case of study of elements used in the industry, it would be wiser to use the vibration methods, where the damage is detected based on the analysis of changes in vibrations that occur when the device is in working order and when damaged. It would be necessary to laminate the piezoelectric elements to measure and control the subsequent oscillations both, passively (without additional excitation) and actively (with additional forcing).

\section{REFERENCES}

1. Białas K. (2010), Passive and Active Elements in Reduction of Vibrations of Torsional Systems, Mechatronic Systems and Materials:Mechatronic Systems and Robotics, Solid State Phenomena, Vol.164, 260-264.

2. Bocian M., Kulisiewicz M. (2013), Method of identifying nonlinear characteristic of energy dissipation in dynamic systems with one degree of freedom, Archives of Civil and Mechanical Engineering, Vol. , 14 (3), pages 354-359.

3. Buchacz A., Wróbel A. (2010), Computer-aided analysis of piezoelectric plates, Solid State Phenomena, Vol. 164, $239-242$.

4. Buchacz A., Banaś W., Płaczek M. (2015), Influence of the excitation parameters of the mechanical subsystem on effectiveness of energy harvesting system, IOP Conf. Series: Materials Science and Engineering, 95, pages 1-6.

5. Buchacz A., Płaczek M. (2014), Modelling of passive vibration damping using piezoelectric transducers - the mathematical model, Eksploatacja i Niezawodnosc, Vol. 16 (2), 301-306.

6. Buchacz A., Płaczek M., Wróbel A. (2013), Control of characteristics of mechatronic systems using piezoelectric materials, Journal of Theoretical and Applied Mechanics, Vol. 51, 225-234.

7. Dymarek A., Dzitkowski T. (2005), Modelling and synthesis of discrete - continuous subsystems of machines with damping, Journal of Materials Processing Technology, Vol. 164-165, 1317-1326.

8. Jamroziak K., Kosobudzki M. (2012), Determining the torsional natural frequency of underframe of off-road vehicle with use of the procedure of operational modal analysis, Journal of Vibroengineering, Vol. 14(2), 472-476.

9. Klarecki K., Hetmańczyk M., Rabsztyn D. (2015), Influence of the selected settings of the controller on the behavior of the hydraulic servo drive, Mechatronics - Ideas for Industrial Application. Advances in Intelligent Systems and Computing, Vol. 317, 91-100.
10. Ociepka P., Herbuś K., Gwiazda A. (2014), Application of the method basing on engineering knowledge and experience for adding the hexapod design process, Advanced Materials Research, Vol. 1036, 1005-1010.

11. Płaczek M. (2012), Dynamic characteristics of a piezoelectric transducer with structural damping, Solid State Phenomena, Vol. 198, 633-638.

12. Płaczek M. (2015), Modelling and investigation of a piezo composite actuator application, Int. J. Materials and Product Technology, Vol. 50(3/4), 244-258.

13. Płaczek M., Wróbel A., Baier A. (2015), Computer-aided strength analysis of the modernized freight wagon, IOP Conf. Series: Materials Science and Engineering, 95, pages. 1-6.

14. Szczepanik M., Stabik J., Wróbel G., Wierzbicki Ł. (2008), The use of thermal imaging systems for testing of polymeric materials, Modelowanie Inżynierskie, 5(36), 279-286 (in Polish).

15. Tuma J., Mahdal M., Suranek P. (2013), Simulation Study of the non-colocated control of a cantilever beam, Mechanics and Control, Vol. 32, 110-116.

16. Tuma J., Simek J., Skuta J., Los J. (2013), Active vibration control of journal bearings with the use of piezoactuators, Mechanical Systems and Signal Processing, Vol. 36, 618-629.

17. Tuma T., Suranek P., Mahdal M., Babiuch M. (2014), Simulation of the parametric excitation of the cantilever beam vibrations, 15th International Carpathian Control Conference (ICCC), Velke Karlovice, Czech Republic.

18. Wróbel A. (2012), Kelvin Voigt's model of single piezoelectric plate, Journal of Vibroengineering, Vol. 14(2), 534-537.

19. Wróbel A.(2015), Analysis of possibility of applying the PVDF foil in industrial vibration sensors, IOP Conf. Series: Materials Science and Engineering, 95, pages 1-5.

20. Wróbel A., Płaczek M., Buchacz A., Majzner M. (2015), Study of mechanical properties and computer simulation of composite materials reinforced by metal, Int. J. Materials and Product Technology, Vol. 50(3/4), 259-275.

21. Wróbel A., Langer P. (2015), Designing of robotic production lines using CAx software, IOP Conf. Series: Materials Science and Engineering, 95 (2015), pages 1-6.

The work was carried out under the project number PBS2/A6/17/2013 agreement implemented under the Applied Research Program, founded by the National Centre for Research and Development. 